\title{
SWAG: Survey of Water and Ammonia in the Galactic Center
}

\section{Jürgen Ott ${ }^{1}$, David S. Meier ${ }^{2}$, Nico Krieger ${ }^{3}$, Matthew Rickert ${ }^{4}$ and the SWAG team}

\author{
${ }^{1}$ National Radio Astronomy Observatory, Socorro, NM, USA, email: jott@nrao.edu \\ ${ }^{2}$ New Mexico Institute of Mining and Technology, Socorro, NM, USA \\ ${ }^{3}$ Max-Planck-Institut für Astronomie, Heidelberg, Germany \\ ${ }^{4}$ Northwestern University, Evanston, IL, USA
}

\begin{abstract}
SWAG ("Survey of Water and Ammonia in the Galactic Center") is a multi-line interferometric survey toward the Center of the Milky Way conducted with the Australia Telescope Compact Array. The survey region spans the entire $\sim 400$ pc Central Molecular Zone and comprises $\sim 42$ spectral lines at pc spatial and sub-km/s spectral resolution. In addition, we deeply map continuum intensity, spectral index, and polarization at the frequencies where synchrotron, free-free, and thermal dust sources emit. The observed spectral lines include many transitions of ammonia, which we use to construct maps of molecular gas temperature, opacity and gas formation temperature (see poster by Nico Krieger et al., this volume). Water masers pinpoint the sites of active star formation and other lines are good tracers for density, radiation field, shocks, and ionization. This extremely rich survey forms a perfect basis to construct maps of the physical parameters of the gas in this extreme environment.
\end{abstract}

Keywords. ISM: molecules, Galaxy: center, ISM: structure, stars: formation

\section{Introduction}

The Galactic Center, being the closest galactic nucleus at a distance of only $8.5 \mathrm{kpc}$, contains $\sim 10 \%$ of the entire Galactic molecular gas within a $\sim 500$ pc region known as the Central Molecular Zone (CMZ). This gas is subject to a unique and extreme environment that is dominated by shocks from cloud-cloud collisions, large fluxes of UV photons and cosmic rays, turbulence, as well as strong magnetic and tidal fields. These conditions are reflected in extreme physical parameters: large temperatures, densities, dispersions, and ionization fractions of the gas, and in a peculiar chemistry that is enhanced in abundances of complex molecules. Similar conditions are also found in high-z galaxies at cosmic times when the bulk of stars were created. To understand the astrophysics of star formation processes in this environment, one must have accurate measurements of these ISM conditions, as all of these physical parameters determine the capability of the molecular gas to cool, collapse, and eventually to form stars. Of particular importance is the temperature of the gas, as it is a direct indicator of the efficiencies of shielding and cooling.

\section{SWAG}

"The Survey of Water and Ammonia in the Galactic Center" (SWAG; survey page at: https://sites.google.com/site/atcaswag) is an approach to measure some of the most important quantities in the CMZ. SWAG data consists of $\sim 42$ spectral lines in the $21-26 \mathrm{GHz}$ range with $4 \mathrm{GHz}$ of continuum at $\sim 0.8 \mathrm{pc}\left(20\right.$ ") spatial and $0.4 \mathrm{~km} \mathrm{~s}^{-1}$ 

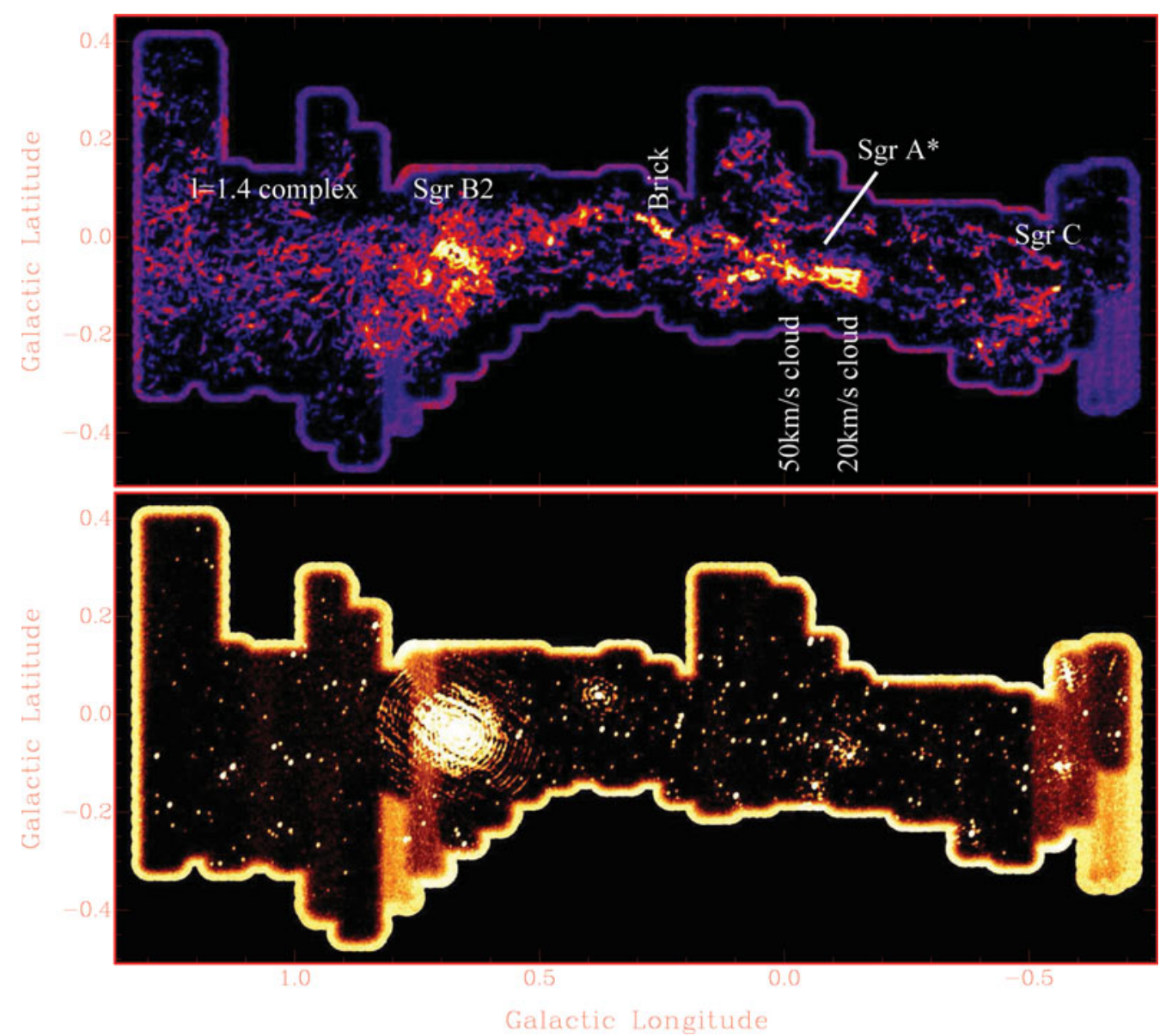

Figure 1. Top: Ammonia (3,3) peak flux map from the first two years of SWAG. Bottom: $22 \mathrm{GHz}$ water maser peak flux map. Both maps cover $\sim 2 / 3$ of the final SWAG area.

spectral resolution. The data are a good compromise between spatial resolution and surface brightness sensitivity, but the interferometric observations do filter out the diffuse gas on the largest scales. The maps therefore reveal the clumpy, filamentary structures of the CMZ in great detail with little confusion.

The SWAG lines include multiple ammonia transitions (Fig. 1 top), which are being used as a thermometer, structure, and dynamic tracer for the molecular gas. Water masers (Fig. 1 bottom) are visible at sites where young stars currently form (in addition to envelopes of evolved stars). We also obtain a rich dataset of density, photo-dominated region and shock tracers (including $\mathrm{c}^{-} \mathrm{C}_{3} \mathrm{H}_{2}, \mathrm{HNCO}, \mathrm{CH}_{3} \mathrm{OH}$ ), radio recombination lines to map the ionized components, and continuum maps to measure the strength, spectral index, and polarization of the emission. Together, SWAG is a comprehensive survey to couple the physical state (density, temperature, ionization, shocks, magnetic field strength) of the molecular gas with the dynamical structure of each cloud embedded in the galactocentric streams. SWAG will also reveal the heating/cooling balance and chemistry of the gas in diverse environments and relates those parameters to each cloud's evolutionary stage of star formation. 